\title{
Déclarer, proclamer : une politique virtuelle
}

\author{
Élise Lamy-Rested
}

Université Libre de Bruxelles et Collège International de Philosophie

elise.lamy.rested@gmail.com

Date de réception : 12-10-2020

Date d'acceptation : 6-12-2020

\section{Résumé}

En suivant le fil conducteur du concept de virtualité dans la pensée derridienne, cet article montre comment, chez Derrida, tout acte politique peut être pensé comme un acte virtuel. La déconstruction du concept de virtualité à partir des Spectres de Marx (1993) permet tout d'abord de comprendre différemment son irruption dans Otobiographies (1984), contemporaines du Séminaire La vie la mort récemment publié (2019) dans lequel on en retrouve le chapitre 2 "Logique de la vivante ", et de lire avec un regard neuf "Du droit à la justice ", la première partie de Force de loi (1993). Comment faire pour que chaque "sur-vie " compte et pas seulement la vie de l'homme blanc qui a rédigé et signé, au nom d'un soi-disant " peuple libre ", la déclaration d'indépendance des Etats-Unis ou des Amériques du sud?

Mots clés: spectralité ; sur-vie ; déconstruction ; déclaration ; peuple ; signature ; justice

\section{Abstract. To declare, to proclaim: A virtual politics}

Following the thread of the concept of virtuality in Derrida's thinking, this article shows how, in Derrida, any political act can be thought of as a virtual act. The deconstruction of the concept of virtuality from Spectres de Marx (1993) allows first of all to understand differently its irruption in Otobiographies (1984), contemporaneous with the recently published seminar La vie la mort (2019) in which we find chapter 2 "Logique de la vivante", and to read with new eyes "Du droit à la justice", the first part of Force de loi (1993). How can we ensure that every "over-life" counts and not only the life of the white man who wrote and signed, in the name of a so-called "free people", the declaration of independence of the United States or South America?

Keywords: spectrality; survival; deconstruction; declaration; folk; signature; justice

\section{Sommaire}

1. De la virtualité à la spectralité

2. La Déclaration d'indépendance: une politique virtuelle?
3. Une politique virtuelle : une politique de la réinvention

Références bibliographiques 
Je parlerai donc de la déclaration d'indépendance comme d'une politique virtuelle, m'engageant ainsi dans une foule de contradictions apparentes dont l'explicitation progressive - à défaut de leur résolution -, me conduira à déconstruire le caractère supposé " juste » des déclarations d'indépendance des Amériques, qu'elles soient du Nord ou du Sud.

"Une politique virtuelle », voici une expression problématique que Derrida n'utilise à ma connaissance pas, ou plus exactement qu'il n'utilise assurément pas dans les deux textes dont je voudrais principalement parler ici. Le premier est "Déclarations d'indépendance ", une conférence qui fut prononcée en 1976 à l'Université de Virginie aux États-Unis et qui constitue la première partie du livre Otobiographies. Contemporain du séminaire La vie la mort, dispensé à l'ENS dans le cadre d'une préparation à l'agrégation, son deuxième chapitre, "Logique de la vivante ", est repris presque à l'identique dans la deuxième séance de ce séminaire. Pour Derrida, la question politique est donc aussi, voire même surtout, une question vitale : il en va de la valeur ou de la justice que l'on doit à la vie, ou plus exactement à la sur-vie, de chaque singularité. On ne s'étonnera donc pas que le second soit "Du droit à la justice " qui ouvrit le colloque donné à la Cardozo Law School en 1989. Si le terme "virtuel " n'apparaît qu'une seule fois dans la deuxième partie d'Otobiographies (Derrida, 1984 : 41) dans un sens déjà déconstruit, il hante tous les Spectres de Marx, même s'il n'est jamais directement ou explicitement attribué au politique. Le " virtuel ", dans Spectres de Marx, concerne plus largement l'espace politique : "Nous tentons ici de ré-affirmer l'héritage de Marx en l'accordant ou en le pliant à une pensée du spectral qui prenne en compte, en particulier dans l'appréhension politique de la chose publique et de son espace (plus ou moins nouveau), une irréductible virtualité » (Derrida, 1993 : note du bas de la page 225). Quoi qu'il en soit, et indépendamment de Derrida, l'expression " politique virtuelle " pourrait sembler contradictoire si l'on définit, de manière très classique, la politique comme un vivre ensemble fondé et soudé par des actes - qui sont autant de lois —, bénéficiant d'une effectivité et d'une réalité immédiate ; et le virtuel comme tout ce qui, étant en puissance, s'oppose à ce qui est en acte. Si le politique puise sans doute ses racines dans ce qui est d'abord en puissance, il n'en demeure pas moins que toute politique a nécessairement pour ultime objectif l'accomplissement de sa finalité quelle qu'elle soit. Une " politique virtuelle ", et destinée à le rester, n'est donc pas simplement une forme politique inaboutie. Elle est beaucoup plus sûrement une contradiction dans les termes. "Une politique virtuelle " serait donc, tout du moins logiquement, un impossible. Pourtant, je crois que c'est bien ainsi que Derrida pense tout acte politique, à savoir comme un acte virtuel, ce qui ne détruit ni son effectivité, ni son actualité, ni même sa capacité à inventer ou à laisser arriver l'événement. C'est ainsi vers un autre sens, plus contemporain et peut-être inédit de la virtualité que nous conduit Derrida. Mais avant d'en déconstruire le sens, Derrida a commencé à penser la virtualité de manière très classique, sans lui faire encore subir cette subversion idiomatique à la déconstruction qui commence toujours par incorporer les concepts traditionnels de l'histoire de la philosophie. 


\section{De la virtualité à la spectralité}

Dans le chapitre 3 de la deuxième partie de De la grammatologie, Derrida analyse le concept de virtualité tel qu'il est utilisé par Jean-Jacques Rousseau dans L'Essai sur l'origine des langues. Selon Rousseau, relu par Derrida, la virtualité concerne l'ensemble des facultés innées qui attendent d'être actualisées par l'imagination ${ }^{1}$, elle-même définie comme la plus active des facultés virtuelles ${ }^{2}$. L'imagination est ainsi la faculté reine qui permettra à l'homme non seulement d'actualiser sa pitié ainsi que toutes ses autres facultés désirantes, mais aussi d'étendre ses désirs à l'infini. La nature n'est donc pas pensée comme " un donné " ou " comme une présence actuelle, mais comme une réserve " (Derrida, 1967 : 263), qui peut être excédée — ou s'excéder elle-même quand les facultés innées de l'individu se laissent emporter par une imagination par définition illimitée et qui est à ce titre une faculté tout à la fois naturelle et surnaturelle. Cette représentation d'une nature déclinable en un ensemble de facultés virtuelles que l'imagination peut pervertir en les disjonctant de leurs limites naturelles, permet à Rousseau d'introduire son concept de perfectibilité qui caractérise le propre de l'homme en le différenciant de l'animal. Mais cette hésitation entre ce que Derrida appelle " une logique du supplément " (l'imagination est non seulement en excès sur la nature, mais elle se substitue à elle en rendant les signes et les apparences plus réels qu'elle ne l'est) et une logique de l'actualisation révèle, selon Derrida, l'attachement de Rousseau à la métaphysique classique et son incapacité à s'arracher à la présence, à la philosophie de la représentation consciente et de l'anticipation, qui appréhende l'histoire comme un sens. L'irruption du concept de virtualité sous la plume de Rousseau, n'aurait en effet d'autre fonction que celle de supprimer le jeu du supplément, incalculable parce que toujours en excès sur la représentation, au profit d'une appréhension aristotélicienne du devenir. Aristote fut en effet le premier philosophe à thématiser de manière rigoureuse le concept de virtualité. Plus exactement, il fut le premier à penser la différence (et donc la relation) entre " les choses en puissance » et " les choses en acte ». Dans le livre 2 de La Physique et dans Métaphysique, livre Thêta, Aristote définit ainsi " ce qui est en puissance " (dynamis) en l'opposant à " ce qui est en acte " (energeia). Ce qui est en puissance est un possible qui attend son actualisation, tandis que ce qui est en acte est effectif, présent et pleinement réalisé. Le passage de la puissance à l'acte est mouvement ou devenir. La chose parvenue à sa forme finale, est enfin dite en acte en ce qu'elle a réalisé son essence. L'acte précède donc la puissance par le temps et par la génération : en s'actualisant, la chose ne fait qu'atteindre sa forme finale qui est en vérité au principe de son mouvement. Par exemple, le bloc de marbre contient en puissance la statue

1. Sur ce point, voir Derrida (1967: 263) : « ... on peut suivre partout, dans l'œuvre de Rousseau, une théorie de l'innéité comme virtualité ou de la naturalité comme potentialité sommeillante. " Derrida souligne.

2. Derrida (1967: 264) : « ...l'imagination qui excite les autres facultés virtuelles n'en est pas moins elle-même une faculté virtuelle : 'la plus active de toutes' ". 
qui est la chose en acte ${ }^{3}$. En d'autres termes, l'avenir est prédéterminé par une essence, ce qui permet de penser une origine et de limiter le jeu du hasard : «Le mouvement s’inscrit dans une logique causale déterminée par la forme finale que chaque chose doit atteindre. Toute la philosophie aristotélicienne du temps et du mouvement s'inscrit ainsi entre une archie et un telos qui constituent les deux pôles du sens ou de l'histoire comme sens ${ }^{4}$. Pensé dans son rapport caché à la logique du supplément, le concept de virtualité (comme toute la problématique de la puissance et de l'acte) a sans doute pour fonction, chez Rousseau en particulier et dans la métaphysique en général, de prédéterminer systématiquement le devenir comme production et développement, évolution ou histoire, en substituant l'accomplissement d'une dynamis à la substitution d'une trace, l'histoire pure au jeu pur, et, comme nous le notions plus haut, une soudure à une rupture. Or le mouvement de la supplémentarité semble échapper à cette alternative et permettre de la penser " (Derrida, 1967 : 265-266).

Mais Derrida n'en reste pas à cette compréhension de la virtualité qu'il va bientôt déconstruire et réinventer dans les Spectres de Marx, en s'inspirant peut-être pour une part (car Derrida ne le dit jamais explicitement) de la philosophie de Deleuze dans laquelle la virtualité joue un rôle clé. Ce concept de virtualité, Deleuze le pense d'abord avec Bergson et en fait l'un des concepts les plus importants de sa philosophie dans Le bergsonisme, avant de le reprendre à son propre compte dans Différence et répétition (voir plus spécialement les pages 269-276). Le coup de force de Deleuze consiste à arracher la problématique du virtuel à l'ontologie et à la temporalité de la présence. Si chez Aristote et dans la métaphysique classique, le virtuel est un possible qui attend sa réalisation, le virtuel devient chez Deleuze une force pleinement réelle ou effective qui ne s'oppose qu’à l'actuel, c'est-à-dire à une réalité présente. Le virtuel est

3. Il faut néanmoins préciser que si le concept de "virtualité » ou de "virtuel » est classiquement rapporté à Aristote (et Derrida ne procède pas autrement dans ce passage de De la grammatologie), ce terme trouve en fait son origine étymologique dans le latin "virtus " qui signifie "force ", "virtualis " en étant un dérivé. Le "virtuel » est ainsi le terme français provenant de la traduction en latin par les philosophes scolastiques du concept grec de dynamis. Le virtuel est chez les scolastiques, tout ce qui, étant en puissance, s'oppose à la réalité en acte.

4. Ce que Derrida réaffirmera dans son article «Ousia et Grammè, note sur une note de Sein und Zeit ", in Marges de la philosophie : "...le couple aristotélicien temps-mouvement est pensé à partir de l'ousia comme présence. L'ousia comme energeia par opposition à la dynamis (mouvement, puissance) est présence. Le temps, qui comporte du déjà-plus et du pas-encore, est un composé. L'énergie y compose avec la puissance. C'est pourquoi il n'est pas, si l'on veut, " en acte ", et c'est pourquoi il n'est pas ousia (étant si l'on veut, subsistant ou substantiel). La détermination de l'étantité (ousia) comme energeia ou entelekheia, comme acte et fin du mouvement est inséparable de la détermination du temps. Le sens $\mathrm{du}$ temps est pensé à partir du présent, comme non-temps. Et il ne peut en être autrement ; aucun sens (en quelque sens qu'on l'entende, comme essence, comme signification du discours, comme orientation du mouvement entre une archie et un telos) n'a jamais pu être pensé dans l'histoire de la métaphysique autrement qu’à partir de la présence et comme présence. » p. 58 - Derrida souligne. 
ainsi une réalité sans présence. Dans les termes de Deleuze, « le virtuel ne s'oppose pas au réel, mais seulement à l'actuel. Le virtuel possède une pleine réalité, en tant que virtuel." (Deleuze : 1968 : 269). Autrement dit la non présence du virtuel n'a aucune incidence sur sa réalité ou son effectivité. Le fait qu'on ne puisse pas se le représenter n'implique pas qu'il n'ait pas d'existence. Le virtuel s'inscrit dans une temporalité échappant à celle - chronologique - de la conscience qui appréhende le réel à partir du possible défini comme l'éventualité pour un événement de, ou de ne pas, se produire. Dans une telle perspective, le réel n'est que le résultat d'une limitation et d'une sélection des possibles. Parmi l'ensemble des possibles concevables, le ou les possibles réalisés sont bien ceux qui n’ont pas été éliminés ou limités dans leur capacité à devenir réels pour des raisons logiques ou ontologiques. Le réel doit ressembler au possible, à tel point que Deleuze, à la suite de Bergson, en vient à soupçonner que c'est en vérité le réel qui donne, après-coup, naissance à un possible en fait totalement imprévisible. Le monstre, qu'Aristote qualifie d'erreur de la nature ou de l'irruption d'une contradiction dans la finalité, serait ainsi l'événement inattendu rendant compte d'un possible incalculable. Ce renversement de l'ordre entre le possible et le réel est aussi un renversement de la métaphysique classique qui s'organise entre une origine ou une essence, et un telos. Si le réel n'est pas un descendant du possible de la métaphysique, c'est parce que le réel n'est pas déterminé par une essence dont la pleine réalisation dépend de son actualisation. Le réel en acte n'est donc soumis à aucune finalité, ce qui permet de ne plus le penser dans son rapport nécessaire au possible onto-chrono-logique. Une telle redéfinition du virtuel, du réel et de l'actuel a au moins deux conséquences. D'une part, l'actuel ne ressemble pas au virtuel qui, bien que réel, demeure inconnaissable et irreprésentable ; d'autre part, l'actuel - en ce qu'il n'est plus déterminé par une fin - reste inachevé, monstrueux, transformable et déformable, c'est-à-dire travaillé par le virtuel. Si le virtuel s'actualise selon une dynamique créatrice et imprévisible, l'actuel se virtualise selon cette même dynamique.

L'usage derridien du virtuel dans « La logique vivante » (Derrida, $1976: 41$ ) puis, plus massivement et plus explicitement, dans les Spectres de Marx, s'inscrit assurément dans cet élan impulsé par Deleuze après sa lecture de Bergson, même si Derrida le repense de manière très singulière. Dans les Spectres de Marx, le virtuel est noué à la spectralité, à l'apparition d'un simulacre, d'une image synthétique ou encore prothétique (Derrida, 1993 : 93). À la différence de Deleuze, Derrida ne soustrait donc pas totalement le virtuel à la représentation, ce qui lui permettra d'articuler les définitions philosophique et technologique du virtuel ${ }^{5}$. À partir des années 1980, le "virtuel » fait en effet son apparition dans le vocabulaire des nouvelles technologies. Il est la sensation de réalité produite chez un sujet par un codage informatique. On parle de "réa-

5. Dans "Artefactualités ", le premier entretien avec Bernard Stiegler d'Échographies de la télévision, entretiens filmés, Derrida propose clairement une définition du virtuel totalement dégagée des problématiques de la puissance et de l'acte, p. 14. 
lité virtuelle " lorsque tous les sens du sujet totalement immergé dans une production numérique, sont sollicités et qu'il est en mesure d'interagir avec elle. La réalité virtuelle n'est donc pas à proprement parler une simple fiction. Elle est plus exactement un double de la réalité ou une réalité d'un autre type qui a tout autant d'effectivité et qui impacte tout autant les sens et le psychisme que la réalité non numérique. Aujourd'hui, l'adjectif virtuel est attribué à toutes les productions des nouvelles technologies, dès lors qu'elles s'inscrivent dans un espace et un temps entièrement créés par le numérique. On parle ainsi de " communication virtuelle ", de " communauté virtuelle " ou encore de " capitaux virtuels ». Dans la philosophie derridienne, le virtuel des nouvelles technologies s'inscrit d'abord dans la dynamique de la survie ${ }^{6}$. Tout être vivant est d'abord un survivant : mortel, il aspire à résister à sa mort pour se garder en vie dans l'ici-maintenant, et pour se garder en sur-vie au-delà de lui-même, en transmettant aux générations futures son savoir scientifique et, si possible, un éclat de sa singularité. Pour rester en sur-vie, le survivant doit pouvoir inscrire son savoir ou cet éclat singulier dans un supplément technique qui, seul, a la capacité de se détacher de son géniteur pour se transmettre de génération grâce à sa capacité à se répéter et à se laisser incorporer par une singularité. Il en va ainsi du signifiant par exemple ou encore de l'image numérique, qui, l'un comme l'autre, véhiculent une foule de spectres, puisqu'ils portent en leur sein et maintiennent en survie une multitude de morts. Si le supplément technique accède à sa représentation consciente lorsqu'il est érigé au rang de signifié, il garde en lui la marque de l'autre de la conscience, une re-présentation qui se répète compulsivement sans jamais se cristalliser dans une identité de pensée ou de perception. La représentation consciente ou actuelle ne ressemble donc en rien à la re-présentation virtuelle qui reste inaccessible, même si elle peut partiellement faire irruption en fracturant la représentation consciente au sein de laquelle elle est enkystée. La représentation actuelle n'a enfin pas plus de réalité que la re-présentation virtuelle, elle n'en est ni isolée ni indépendante. Toute représentation actuelle est travaillée par une re-présentation virtuelle et est en voie de virtualisation. De même toute re-présentation virtuelle est en voie d'actualisation, sans quoi elle ne pourrait faire effraction dans notre ici-maintenant. Le développement fulgurant des technologies ou des " télé-technologies " dans les termes de Derrida (Derrida, 1993 : $268)^{7}$ est le symptôme de cette dynamique de la survie qui crée un espace-temps radicalement différent de celui de la conscience ou de l'ici-maintenant. Le temps virtuel est celui du différé ou de l'espacement : le supplément technique n'est pas destiné aux vivants. Mais il est aussi celui de l'immédiateté ; la répétition est ponctuelle, compulsive, effrénée et jamais identique à elle-même. L'espace virtuel est tout à la fois étendu jusqu’à un inconnaissable qui excède la

6. Je n'aurai pas l'opportunité de développer ici cette question de la survie. Pour un tel développement et une explicitation de cette problématique, je renvoie à Lamy-Rested (2017).

7. Le préfixe " télé " fait référence au telos grec. Sous la plume de Derrida, " télé » (loin de...) désigne aussi l'espacement. 
représentation consciente, et tout entier réduit dans un instant qui est celui de l'irruption intempestive et compulsive d'un spectre : "Mais il y va aussi, indissociablement, du déploiement différantiel de la tekhnè, de la techno-science ou de la télé-technologie. Il nous oblige plus que jamais à penser la virtualisation de l'espace et du temps, la possibilité d'événements virtuels dont le mouvement et la vitesse nous interdisent désormais (plus et autrement que jamais car ce n'est pas absolument et de part en part nouveau) d'opposer la présence et sa représentation, le " temps réel " au " temps différé ", l'effectivité à son simulacre, le vivant au non-vivant, bref le vivant au mort-vivant de ses fantômes. Il nous oblige à penser à partir de là, un autre espace pour la démocratie. Pour la démocratie à venir et donc pour la justice. » (Derrida, 1993 : 268).

La Déclaration d'indépendance, comme tout acte politique - peu importe qu'il soit juste ou non - est donc bien un acte virtuel. Comment penser cette apparente contradiction ? Pourquoi la politique est-elle, selon Derrida, nécessairement virtuelle ? Et que faire enfin d'une politique virtuelle?

\section{La Déclaration d'indépendance : une politique virtuelle?}

Déclarer, proclamer sont des actes de langage ou plus précisément des performatifs dans les termes d'Austin qui, à certaines conditions, peuvent devenir effectifs, c'est-à-dire avoir un impact sur le réel ou le modifier. Si Austin n'avait sans doute pas en tête l'idée que son performatif pourrait un jour être soupçonné d'être virtuel sans ne rien perdre de son effectivité, c'est pourtant bien dans cette apparente aporie que l'entraîne Derrida ${ }^{8}$. La Déclaration d'indépendance qui permet à tel peuple de s'auto-proclammer libre et de se délivrer de la tutelle de telle colonie, est en fait marquée d'une certaine indécidabilité que Derrida associe à une fiction : "Cette obscurité, cette indécidabilité [...] sont requises pour produire l'effet recherché. Elles sont essentielles à la position même d'un droit comme tel, qu'on parle ici d'hypocrisie, d'équivoque, d'indécidabilité ou de fiction " (Derrida, $1984: 21$ ). Pourquoi une telle indécidabilité ? En quel sens est-elle liée à la fiction ? Comment est-il possible de faire de l'indécidabilité et de la fiction des attributs de ce que j'ai appelé une "politique virtuelle"?

Il en va d'abord d'une signature. Pour que la Déclaration d'indépendance puisse être effective, il faut qu'elle soit signée par un peuple, celui qui revendique son indépendance avant de la gagner par l'apposition de sa signature au bas de la Déclaration. Or le peuple ne peut pas, à proprement parler, signer une Déclaration d'indépendance. Celui (puisqu'il n'est jamais question de femmes dans les Déclarations d'indépendance...) ou ceux qui l'ont signée ne sont en fait que les représentants du peuple. La structure même de la démo-

8. Ou plus exactement que je voudrais l'entraîner, car, à ma connaissance, Derrida n'articule jamais explicitement le performatif austinien et le virtuel. Mais en prolongeant sa pensée, il me semble légitime et justifié d'assumer cette articulation. Si la politique est un espace virtuel, sachant qu'elle se décline en une multiplicité d'actes de langage, il faut pouvoir penser la virtualité de ces actes de langage. 
cratie implique donc une série de représentants qui s'expriment " au nom du peuple " : "En signant, le peuple dit - et fait ce qu'il dit faire, mais en le différant par le truchement de ses représentants dont la représentativité n'est pleinement légitimée que par la signature..." (Derrida, 1984 : 22).

Le représentant est ainsi censé se faire l'écho de la voix du peuple et de rédiger sous son autorité. Mais ce peuple, au nom duquel est soi-disant rédigée la Déclaration, n'a en fait aucune existence : "Or ce peuple n'existe pas. Il n'existe pas avant cette déclaration, pas comme tel. S'il se donne naissance, en tant que sujet libre et indépendant, en tant que signataire possible, cela ne peut tenir qu'à l'acte de signature " (Derrida, 1984: 21-22). Autrement dit, la Déclaration d'indépendance est une contradiction dans les termes si l'on en croit la différence établie par Austin entre le constatif qui se contente de rapporter un fait, et le performatif qui a le pouvoir de modifier le réel ${ }^{9}$. Pour qu'une telle Déclaration soit possible, il faut en effet supposer qu'il existe, avant même sa rédaction, un peuple revendiquant son indépendance. Mais simultanément, c'est seulement à l'instant où la Déclaration est signée que le peuple existe. En d'autres termes, la signature de la Déclaration produit ou invente le "peuple libre » qui ne peut donc se reconnaitre en elle, si tout du moins il le peut, que de manière rétroactive. C'est donc après-coup que le soi-disant peuple peut se désigner comme un peuple, alors même qu'il a censément ordonné la rédaction de la Déclaration. La Déclaration est donc non seulement enfermée dans l'indécidabilité (le peuple est produit par la Déclaration alors qu'il en est aussi à l'origine ; autrement dit, la Déclaration crée le peuple autant qu'elle constate son existence), mais elle porte de surcroît en elle la marque d'une fiction qui simultanément jouit d'une certaine effectivité. Si le peuple avant la Déclaration n'existe empiriquement pas, c'est en fait aussi un abus de langage d'affirmer qu'il est produit par la signature de la Déclaration. Le peuple comme unité, le peuple comme sujet libre ou comme "soi » est en fait un fantasme. De facto, la Déclaration n'est signée que par ses Représentants qui ainsi donnent corps à leur fantasme. Sans développer ce point, je rappellerai juste que Derrida s'est toujours méfié du dit " peuple " notamment pour une raison qui intéresse directement mon présent propos : le peuple est associé au communautarisme qui repose sur l'affirmation d'une identité et qui implique la formation d'un "nous " discriminant. Le peuple n'est qu'un mot ou une représentation derrière lesquels se dissimule une multiplicité de singularités, comme Jacques Derrida le rappelle dans son dialogue avec Jean-Luc Nancy et Philippe Lacoue-Labarthe à lire dans la revue Rue Descartes. Le peuple fantasmé des États-Unis de l'année 1776 - puisque c'est de cette Déclaration qu'il s'agit

9. Voir J. L. Austin, Quand dire, c'est faire. Outre la définition du performatif, Austin établit une distinction entre l'énoncé constatif qui rapporte un fait et qui dépend des valeurs "vrai/ faux ", et l'énoncé performatif qui crée un fait nouveau. On évalue la force d'un performatif à sa réussite ou à son échec. Il faut noter qu'Austin lui-même reviendra sur cette distinction beaucoup trop théorique pour épouser la fluctuation et l'ambiguïté du langage ordinaire. Quoi qu'il en soit, Derrida (qui ne cite pas le nom d'Austin dans ce texte), fait comme s'il était l'auteur de cette " indécidabilité » entre le constatif et le performatif. 
dans le texte de Derrida - , exclut par exemple les femmes pour ne rien dire des noirs. Quant aux Indiens qui, bien sûr, ne font pas partie du "peuple libre ", sont-ils seulement considérés comme des habitants légitimes de l'Amérique du nord ? On est bien en droit d'en douter... Ledit " peuple libre » est donc exclusivement blanc et masculin. La signature de la Déclaration ne rend donc pas effectif ou réel un peuple qui n'existait auparavant que potentiellement, mais donne une actualité et une effectivité à un peuple qui reste et qui restera virtuel au sens où il est une représentation supplémentaire (un signifiant cristallisé dans un signifié) dont la réalité ne repose que sur la force d'un crédit et qui reste travaillée par son autre non actuel, non conscient et non présent. Dans les termes de Derrida : "Le soi surgit ici dans tous les cas (nominatif, datif, accusatif) dès lors qu'une signature se fait crédit, d'un seul coup de force, qui est aussi un coup d'écriture, comme droit à l'écriture " (Derrida, 1984 : 23). Autrement dit, la rédaction puis la signature de la déclaration fonde juridiquement et dans la violence un peuple auquel on ne peut que croire. La politique des États-Unis née de cette Déclaration d'indépendance est donc tout entière virtuelle au sens où elle est un fantasme réel. Car cette politique virtuelle a bel et bien réellement imposé ses lois sexistes et raciales sur l'ensemble du territoire américain pendant de nombreuses années. C'est sans doute en ce sens qu'il faut comprendre cet autre passage de "Déclarations d'indépendance " : "On peut entendre cette Déclaration comme un vibrant acte de foi, comme une hypocrisie indispensable à un coup de force politico-militaro-économique, etc. " (Derrida, 1984 : 27). La Déclaration est, en d'autres termes, le résultat d'un geste théologico-politique et ce pas uniquement parce qu'elle est un acte de foi. Le peuple comme unité ou comme soi fantasmé n'a de sens et de consistance qu'en ce qu'il est légitimé par Dieu qui en est l'ultime fondement. S'il est toujours possible de soupçonner le peuple d'être fictif, il est impossible de soupçonner Dieu de l'être. C'est au nom de Dieu que la Déclaration doit donc être signée. Et l'idée de peuple est une répétition ou un substitut de l'idée du Dieu chrétien. Si le peuple forme une unité dont la spécificité est de pouvoir agir, s'il est en d'autres termes un sujet, il ne peut s'exprimer que par le biais de représentants qui incarnent le peuple à l'instar du Christ qui incarna et représenta Dieu sur Terre. C'est donc à une violente critique des Déclarations que Derrida se livre ici, en les réduisant à des textes logocentriques ${ }^{10}$ ou phallocentriques, c'est-à-dire à des textes produits par la conscience quant à elle définissable comme cet acte réfléchissant par lequel on se représente comme un soi ou un sujet pensant.

10. Le logocentrisme est un terme qui apparaît dès Derrida (1967). Par ce terme, Derrida veut désigner l'ensemble de l'histoire de la philosophie qui ne serait qu'une déclinaison de la philosophie platonicienne organisée autour du logos. Le discours rationnel permettrait seul d'accéder à la contemplation des essences et à la vision (ou à la représentation) de l'origine divine de toutes les choses. Le christianisme, que l'on peut très vite définir comme la reformulation du judaïsme dans des concepts grecs enfin traduits en latin et qui est fondé sur la présence de Dieu à travers le Christ, ne serait lui-même que le rejeton de la philosophie platonicienne. 
Pourtant, comme tout texte logocentrique, la Déclaration contient en elle-même les éléments de sa déconstruction. Sa virtualité même est le gage de sa déconstruction et une chance d'invention d'une autre politique, que je voudrais maintenant tenter de penser avec vous. En effet - et je cite encore Derrida - " cette fable implique de la trace et n'est en vérité possible que par l'inadéquation à soi-même d'un présent " (Derrida, 1984 : 23). Pour parler vite, le peuple-fiction introduit une instabilité au cœur même de la Déclaration. Seule la vérité est parfaitement adéquate à elle-même, immuable et atemporelle. Le caractère fictif ou virtuel de la Déclaration ouvre la voie à l'invention d'une autre politique : la politique et la démocratie sont toujours virtuelles non seulement parce qu'elles s'organisent autour d'un système de représentations fictives qui reposent sur la force du crédit, mais aussi parce que chaque politique ou chaque démocratie quelle qu'elle soit reste inventive. C'est donc vers une autre approche du virtuel que je voudrais à présent vous conduire pour penser la " politique virtuelle » qu'aurait peut-être rêvée Derrida. Le virtuel ne sera ni pensé comme cette potentialité qui attend son actualisation, ni seulement comme cette fiction soutenue par la force d'un crédit, qui fait d'elle une présence. En confrontant «Déclarations d'indépendance " à " Du droit à la justice " qui constitue la première partie de Force de $l o i$, j'essaierai de montrer comment la politique virtuelle derridienne est en fait une politique de perpétuelles réinventions. Autrement dit, le virtuel doit alors être entendu comme cette fiction qui, en tolérant la déconstruction ou la mise en suspens de la force du crédit qui la sous-tend, se donne les moyens de perpétuellement se réinventer.

\section{Une politique virtuelle : une politique de la réinvention}

La Déclaration d'indépendance trouve donc son fondement légitime et ultime en Dieu qui, dans la religion Chrétienne, constitue aussi la représentation ultime. La déconstruction de cette ultime représentation, Dieu, sur lequel sont en réalité fondées toutes les démocraties occidentales, même celles qui se réclament de la laïcité ${ }^{11}$, permet de penser un autre droit, une autre justice et une autre politique auxquelles Derrida en appelle dans " Du droit à la justice ». Je n'aurai pas ici le temps de montrer comment Derrida déconstruit cette idée de Dieu, même si «Du droit à la justice » comme l'ensemble de son œuvre s'inscrit vraisemblablement dans cette dynamique de déconstruction de l'idée de Dieu, quelle que soit la religion institutionnelle à laquelle il appartient. Dans « Du droit à la justice », Derrida interroge une expression de Montaigne, reprise par Pascal : «le fondement mystique de l'autorité ». Le droit est avant tout un texte qui détient son autorité de la force du crédit qu’on lui accorde. Suspendre son crédit, c'est simultanément initier le travail de la déconstruction du droit et se donner la chance d'inventer une décision qui ne soit plus en

11. Il serait trop long de développer ce point ici, mais je renvoie à Foi et savoir, dans lequel Derrida montre pourquoi la laïcité est inséparable de la pensée chrétienne. 
parfaite adéquation avec le droit en vigueur. C'est, autrement dit, se donner la chance d'inventer un autre droit. Or cette chance de déconstruction du droit est la justice. Sans chercher à penser ici ce que Derrida entend précisément par justice, et pour rester dans la dynamique de mon propos, je dirai juste que la justice est ce fondement irreprésentable et informulable (ou mystique au sens de Wittgenstein) qui ne peut pourtant se donner que dans cette représentation qu'est déjà la décision, c'est-à-dire dans un acte de langage. Cet acte de langage, tout effectif qu'il soit, n'en demeure pas moins virtuel. Le Réel, que j'écrirai avec un $\mathrm{R}$ majuscule, celui qu'on ne peut pas atteindre mais qui est assurément effectif ${ }^{12}$, est sans doute de l'ordre de la justice et ne peut se donner que dans une représentation simultanément virtuelle et actuelle qui a le pouvoir de transformer la réalité. En la politique, en son droit ou en ses lois, on ne peut que croire et il est toujours possible de la transformer. Parce qu'elle est virtuelle, elle n'est en effet soumise qu'à une seule loi : celle que l'on veut bien lui donner. Cette loi se maintient enfin par la force d'un crédit. Suspendre ce crédit, c'est déjà se donner la chance d'inventer une autre politique qui elle-même sera destinée à être réinventée lorsque le contexte aura changé, puisqu'il n'existe en effet aucun fondement, aucune essence sur lesquels pourrait se construire, en vérité, une politique. Monstrueusement virtuelle, la politique est incalculable et imprévisible.

\section{Références bibliographiques}

(2006). " Dialogue entre Jacques Derrida, Philippe Lacoue-Labarthe et JeanLuc Nancy ». Rue Descartes, 2 (2), 86-99. <https://doi.org/10.3917/rdes.052.0086>

Aristote (1986). Métaphysique. Traduction Jules Tricot. Paris : Vrin.

- (1999). Physique. Traduction Lambros Couloubaritsis et Annick Stevens. Paris : Vrin.

Austin, John Langshaw (1970). Quand dire, c'est faire. Traduction et introduction Gilles Lane. Paris : Seuil.

Deleuze, Gilles (1966). Le bergsonisme. Paris : PUF.

- (1968). Différence et répétition. Paris : PUF.

Derrida, Jacques (1967). De la grammatologie. Paris : Minuit.

- (1972). Marges de la philosophie. Paris : Minuit.

- (1976). Fors, la préface au Le verbier de l'homme aux loups de Nicolas Abraham et Maria Torok. Paris : Flammarion.

- (1984). Otobiographies. L'enseignement de Nietzsche et la politique du nom propre. Paris : Galilée.

- (1993). Spectres de Marx. L'État de la dette, le travail du deuil et la nouvelle Internationale. Paris : Galilée.

— (1994). «Du droit à la justice ». In : Force de loi. Paris : Galilée.

12. Dans Fors, Derrida traite du Réel comme de cette force agissante mais irreprésentable et insaisissable. 
- (1996). Foi et savoir. Paris : Seuil.

- (2019). La vie la mort. Séminaire (1975-1976). Paris : Seuil, bibliothèque Derrida.

Derrida, Jacques et Stiegler, Bernard (1996). Échographies de la télévision, entretiens filmés. Paris : Galilée.

Lamy-Rested, Elise (2017). Excès de vie, Derrida... Paris : Kimé.

Rousseau, Jean-Jacques (1781). Essai sur l'origine des langues. Paris : Garnier Flammarion, 1993.

Elise Lamy-Rested, après une thèse de doctorat sur la philosophie de Derrida, est actuellement postdoctorante à l'Université Libre de Bruxelles (H2020-MSCACOFUND) et directrice de programme au Collège International de Philosophie. Elle travaille sur l'articulation entre vie et technique dans la philosophie. Elle a publié, entre autres, Excès de vie. Derrida... aux éditions Kimé en 2017.

Elise Lamy-Rested holds a Ph.D. in Philosophy specializing in Derrida. She is currently a postdoctoral fellow at the Université Libre de Bruxelles (H2020-MSCACOFUND) and a program director at the Collège International de Philosophie. Her research centers on the articulation between life and technique in contemporary philosophy. In addition to several research articles, she has published the book Excès de vie. Derrida... (Éditions Kimé, 2017). 